\title{
Effects of the Cytotoxic T-Cells on the Dynamics of Co-Infection of HIV-1 and Mycobacterium tuberculosis
}

\author{
Chipo Mufudza, Senelani D. Hove-Musekwa, Edward T. Chiyaka \\ Department of Applied Mathematics, Modelling Biomedical Systems Research Group, National University of Science and Technology, \\ Bulawayo, Zimbabwe \\ Email: chipo.mufudza@nust.ac.zw, senelani.hove-musekwa@nust.ac.zw, edward.chiyaka@nust.ac.zw
}

How to cite this paper: Mufudza, C., Hove-Musekwa, S.D. and Chiyaka, E.T. (2016) Effects of the Cytotoxic T-Cells on the Dynamics of Co-Infection of HIV-1 and Mycobacterium tuberculosis. Journal of Tuberculosis Research, 4, 191-212. http://dx.doi.org/10.4236/jtr.2016.44022

Received: October 28, 2016

Accepted: December 12, 2016

Published: December 15, 2016

Copyright $\odot 2016$ by authors and Scientific Research Publishing Inc. This work is licensed under the Creative Commons Attribution International License (CC BY 4.0).

http://creativecommons.org/licenses/by/4.0/

\begin{abstract}
Enhancement of the Human Immunodeficiency Virus (HIV) specific cytotoxic Tcells mechanisms in an HIV-1 and Mycobacterium tuberculosis (Mtb) co-infected individual seems to improve the clinical picture of an individual by reducing Acquired Immuno Deficiency Syndrome (AIDS) state progression rate. In this paper, we develop a system of deterministic differential equations representing the immune cells involved in an HIV-1 and Mtb co-infected individual. Results show that although the non-lytic arm of the HIV-1 cytotoxic T-cells affects the co-infection dynamics more than the lytic factors, a combination of both factors results in a more positive reduced progression to the AIDS state. This is due to the increased protection of the $\mathrm{CD} 4^{+} \mathrm{T}$-cells by the CTL mechanisms by further reducing infections and replications by the HIV. Thus, HIV-1 specific CTLs mechanisms' involvement is here recommended to be part of a solution to the HIV and Mtb co-infection problems.
\end{abstract}

\section{Keywords}

Mycobacterium tuberculosis, HIV, Co-Infection, Cytotoxic T-Cells, Lytic and Non-Lytic Factors

\section{Introduction}

\subsection{HIV and Mycobacterium tuberculosis (Mtb) Co-Infection}

Since the resurgence of HIV, Mtb and HIV has been closely linked, the HIV-1, Mtb co-infection causes a two-infection disease endangering human immune response which may also differ depending on the genetic background [1]. Approximately one- 
third of the world's population is latently infected with Mtb [2]. The rate of progression from infection to full blown disease varies greatly with at least $10 \%$ of Mtb infected individuals developing clinical disease and about half of them developing disease more than two years after infection, commonly named reactivation or post-primary TB [3]. Thus, the lifetime risk of developing active TB in immunocompetent adults is estimated to be $5 \%-10 \%$, but in HIV-positive individuals this risk is increased to $5 \%-15 \%$ annually [4]. The depletion of $\mathrm{CD} 4^{+}$T-cells, which is a main feature of AIDS, is certainly an important contributor to the increased risk of reactivation of latent $\mathrm{TB}$ and susceptibility to new Mtb infection. HIV also manipulates macrophage bactericidal pathways [5], deregulates chemotaxis [6], and tips Th1/Th2 balance [7] and may impair Tumor Necrosis Factor (TNF)-mediated macrophage apoptotic response to Mtb and thus facilitates bacterial survival [8]. Specifically, TB patients with AIDS present a dominant granulocytic infiltrate and necrosis without the typical necrosis seen in nonHIV-infected TB granulomas due to the killing of $\mathrm{CD} 4^{+} \mathrm{T}$-cells in the granuloma by the HIV [9].

HIV produces a progressive decline in the cell mediated immunity by virtue of the pathogen targeting the $\mathrm{CD} 4^{+} \mathrm{T}$-cells eventually disabling them and making them dysfunctional. The decline impacts negatively on the control of the Mtb by the immune system since the $\mathrm{CD} 4^{+} \mathrm{T}$-cells are also needed for the cell immune mediated response for the Mtb infection to keep TB in check. HIV also alters the pathogenesis of TB, greatly increasing the risk of developing active disease in a co-infected individual leading to more extra pulmonary involvement and radiographic manifestations.

The function of many immune cells, including macrophages and Dendritic cells (DCs), is modulated by both HIV and Mtb, with the presence of Mtb increasing replication of the virus in a co-infected individual [10]. Mtb has been reported to up-regulate HIV-1 replication in chronically or acutely infected T-cells or macrophages [11] [12]. The primary target for Mtb, the alveolar macrophage, can also be infected with HIV exacerbating HIV replication in macrophages and lung cells obtained by bronchoalveolar lavage from co-infected individuals [10] [12] [13]. In-vitro Mtb infection can up-regulate both HIV infection and replication within monocyte-derived macrophages (MDMs), increase the efficiency of virus transmission from infected MDMs to T-cells, and favor replication of X4 HIV variants by upregulation of CXCR4 [14]. It is therefore evident that the presence of each of these diseases has a profound effect on the other due to the interactions with the immune system. This is because the frequency at which HIV and Mtb occurs together is determined by the epidemiology of each of the disease in a given population. Thus, co-infection has also become one of the main complications worldwide with TB being the cause of death for one out of every three people with AIDS.

\subsection{Why Cytotoxic Cells?}

The challenges that the world is facing in HIV and Mtb co-infection can possibly be solved through a combined vaccine. However, design of candidate vaccines is a parti- 
cularly difficult task since laboratory correlates of protection have not been defined for Mtb and HIV infections. Vaccination efforts have shown that Mtb infection diminish HIV-specific Immunoglobulin A (IgA) responses at mucosal surfaces which help prevent HIV infection or reduce the viral load [15]. In addition, vaccine-induced immune responses need to be tipped towards protection, avoiding those that may result in immunopathology, this requires meticulous study of appropriate adjuvants, antigens, and vaccination regimens for the novel vaccines [16]. Even with treatment, it has been noted with serious concern regarding current recommendations for treatment of HIVMtb co-infection since data suggest that at least 8 months duration of rifapentine (RPT), or rifampin (RIF) therapy, initial daily dosing, and concurrent antiretroviral therapy might be associated with better outcomes, but adequately powered randomized trials are urgently needed to confirm [17]. The criteria for deciding between strategies to treat a co-infected individual still remains a challenge although a more robust quantitative measure could help by incorporating rate of change of $\mathrm{CD} 4^{+}$count as a measure of Integrated Risk Information System (IRIS) risk, as well as viral and bacterial load, drug toxicity, and improved measures of drug interaction [18]. The presence of the CTLs cannot be sidelined in an effort to come up with solutions to HIV-Mtb coinfections. Studies have shown that the presence of Mtb affect the C38 expression on the $\mathrm{CD}^{+}$cells responsible for CTL differentiation. This negatively impacts the HIV progression which may also depend on the ethnic group (genes) [19]. There have been several hypotheses set regarding the relationship between HIV specific CTLs and viral load. The findings strongly support the involvement of CTLs in the control of HIV infection [20] but does it have the same effect in the case of co-infection? The CD8 ${ }^{+}$ cellular responses like CTLs are potential mediators of protection against HIV [21].

Understanding the vital effects of Cytotoxic T-cells as an immune system component might be of paramount importance for vaccine development. The tropism of the co-infection between HIV-1, Mtb and the immune system studied by Kirshner [22] can be bridged by including the role of the Cytotoxic T-cells (CTLs: a special type of the $\mathrm{CD}^{+} \mathrm{T}$-cells) in the immune system. CTLs play an important role in controlling both HIV and Mtb. This is made possible by incorporating the lytic and non-lytic responses of the CTLs. The lytic response involves the destruction of infected T-cells by the effector cells. The CTLs are antigen specific as they possess receptor molecules on their surface that can recognise the antigen epitope (portion of antigen which which specifically interacts with the immune system). These receptors are designed in such a way that they recognise antigen only when they are bound to a cell surface molecule called the major histocompatibility complex (MHC). The MHC presents peptides to CTLs which then destroy the infected cell if it can bind the peptide-MHC class1 complex [23]. In a co-infected individual, the CTLs are responsible for killing infected T-cells by both HIV and Mtb. This is made possible when naive $\mathrm{CD}^{+} \mathrm{T}$-cells are converted into CTLs which are either HIV specific CTLs or Mtb specific CTLs through the B-cells. The CTLs are essential in many ways in the control of Mtb infection through their different effector mechanisms including activation of macrophages when they induce the release of the Interferon gamma (INF- $\gamma$ ) thereby by activating infected 
macrophages to induce reactive nitrogen intermediates [24]. They also mediate lysis of infected macrophages through the Fas-FasL pathway predominantly achieved by the $\mathrm{CD}^{+} \mathrm{T}$-cells and through the granule exocytosis pathway [24]. CTLs also induce target cell apoptosis and kill the bacteria directly by the granulysin which result in the alteration of bacterial cell membrane.

In this paper, we focus on the HIV specific CTLs only and how they help to reduce the two infection disease progression. Non-lytic response of the CTLs involves the prevention of infection of new cells and inhibition of replication by soluble mediators secreted by the immune system. The CTLs then caters for the non-lytic response by producing chemokines like the $\beta$-chemokine that helps to reduce viral load at the early stages of the HIV infection (the asymptomatic stage) as they block the entry of virions into the $\mathrm{CD}^{+} \mathrm{T}$-cells [25]. They also produce cytokines. The HIV specific CTLs principally produce the INF- $\gamma$, Interleukin-6 (IL-6) and Interleukin-10 (IL-10) which suppress the rate of viral production [26]. The INF- $\gamma$ cytokines produced by the CTLs are also responsible for the activation of the macrophages so that they clear the Mtb bacteria and the HIV virus. Although we are not going to incorporate the Mtb specific CTLs, it should be noted that they also produce the INF- $\gamma$ cytokine and the TNF which again are responsible for the inhibition of new infection by the Mtb. We will also consider the dynamics looked at by Kirshner [22] and incorporate the HIV specific CTLs mechanisms in the immune system and propose a mathematical model describing the dynamics of uninfected T-cells, virally infected $\mathrm{CD} 4^{+} \mathrm{T}$-cells, macrophages, HIV pathogen, Mtb pathogen and the HIV specific CTLs with the immune system.

\section{Model Formulation}

The model by Kirshner [22] is here introduced and used as our basic model. This model focuses on the study of the hypothesis that the presence of infection of HIV with Mtb in the body worsens the clinical picture of HIV and that the presence of HIV can activate the Mtb infection. This was done incorporating the interactions of the immune system's key players which include the lymphocytes or T-cells, macrophages, HIV and Mtb. Four population groups of cells and pathogens are here used namely: the armed $\mathrm{CD}^{+}$and $\mathrm{CD}^{+}$T-cell population at time $t$ represented by $T(t)$, the macrophage population at a given time represented by $M(t)$, the HIV population is represented by $V(t)$ and the Mtb population at a time $t$, represented by $T_{b}(t)$. The model is explained in detail in [22]. The following system of equations were proposed:

$$
\begin{aligned}
& \dot{T}(t)=s_{T}-\mu_{T} T(t)+r_{t}\left(\frac{V(t)+T_{b}(t)}{C+V(t)+T_{b}(t)}\right)-k_{1} V(t) T(t), \\
& \dot{M}(t)=\mu_{M}\left(M_{0}-M(t)\right)-k_{2} M(t) V(t)+r_{2 m} M(t) V(t)+r_{1 m} M(t) T_{b}(t), \\
& \dot{V}(t)=V(t)\left(N_{1} k_{1} T(t)+N_{2} g_{v} M(t)\right)-V(t)\left(k_{3} T(t)+k_{4} M(t)\right)-\mu_{v} M(t), \\
& \dot{T}_{b}(t)=r_{T_{b}} T_{b}(t)\left(K-T_{b}(t)\right)-\mu_{T_{b}} T_{b}(t)-T_{b}\left(k_{5} T(t)+k_{6} M(t)\right) .
\end{aligned}
$$


The interactions of both pathogens with the immune system are explored in [22] using system (1) and results show that the T-cells population is lower in the presence of both pathogens than in the case of HIV alone. The results also showed that viral load is higher in a co-infected patient than in a single infected patient and the same trend was observed for the Mtb. Thus, since progression to AIDS is based on the CD4 $4^{+} \mathrm{T}$-cell count and the viral load, the presence of Mtb in an HIV infected individual worsens the clinical picture of the AIDS state.

\section{Model with CTL Mechanisms}

The lytic and non-lytic factors of the HIV specific CTLs are here incorporated to system (1), in order to analyse their effects to the dynamics of disease progression in a co-infected individual. In particular, HIV specific CTL T-cells response in HIV infection and its effects to the whole immune system dynamics. Thus CTLs are here modelled with their HIV specific function and not just as general T-cells as done by [27] and by monitoring the dynamics of six groups of population cell densities: the population density for the uninfected T-cells at a time $t$ (a pool of the $\mathrm{CD} 4^{+} \mathrm{T}$-cells, Mtb specific CTLs and naive CD8 ${ }^{+} \mathrm{T}$-cells) $T(t)$; the virally infected $\mathrm{CD} 4^{+} \mathrm{T}$-cells at a time, $T^{*}(t)$; the free viral particles population at a time, $V(t)$; the density of the resting, activated and infected macrophages, $M(t)$; the population density of the Mtb pathogen at a time, $T_{b}(t)$ and the density of the HIV-1 specific CTLs population at a time $t, C(t)$. We also assumed that the immune cells proliferation has different limit values since their production is controlled by the source from which they are coming from either the thymus for the $\mathrm{CD} 4^{+} \mathrm{T}$-cells and the $\mathrm{CD} 8^{+} \mathrm{T}$-cells and the bone marrow for the macrophages. Proliferation of cells is treated differently in the model depending on whether infection is by the virus or the Mtb bacteria.

Uninfected $\mathrm{CD}^{+} \mathrm{T}$-cells are produced from the thymus at a rate of $s_{T}$ and die naturally at a rate $\mu_{T}$. Proliferation of the T-cells due to infection by both the Mtb and HIV pathogens are modelled by the factors $r_{m}\left(\frac{T_{b}}{T_{b}+D_{T_{b}}}\right)$ and $r_{t}\left(\frac{V}{V+D_{v}}\right)$ respectively, where $r_{t}, r_{m}$ are maximum proliferation rates and $D_{T_{b}}, D_{v}$ are parameters that determine the amount of pathogen needed for half maximum stimulation of CD $4^{+}$ T-cells by the Mtb and HIV respectively [22]. Parameter $k_{v}$ represents the apoptotis with $B_{T}$ being the level of apoptotic engagement by the receptors [27]. Infection of the $\mathrm{CD} 4^{+} \mathrm{T}$-cells and the macrophages by HIV occurs at rates $k_{1}$ and $k_{2}$, respectively, with the infection haboured due to the presence of the CTLs by a factor of $\left(\frac{1}{1+a_{0} C(t)}\right)$, where $a_{0}$ is the efficiency of each CTL in reducing $\mathrm{CD} 4^{+} \mathrm{T}$-cell infection [27]. Infected CD4 ${ }^{+}$T-cells are also directly killed by the CTLs at a rate $h$ and lost due to bursting and natural death at a rate of $\alpha$ releasing $\alpha N_{T}$ and $\alpha_{m} N_{m}$ virions from the infected $\mathrm{CD} 4^{+} \mathrm{T}$-cells and macrophages, respectively. Again, this viral replication is limited due to the presence of the CTLs as they produce chemokines by a 
factor of $\left(\frac{1}{1+b_{0} C(t)}\right)$, where $b_{0}$ is the rate at which CTLs suppresses viral production. Macrophage proliferation due to infection by the HIV and Mtb are modeled at the rates $r_{1 m}$ and $r_{2 m}$, respectively. Mtb pathogen can also be eliminated from the system by phagocytosis action of Mtb specific CTLs (here just considered as T-cells) at a rate $k_{3}$ or by the macrophages at rate $k_{4}$. The logistic growth rate of the Mtb bacteria is accounted for by $r_{T_{b}} K T_{b}$, where $K$ as carrying capacity and the pathogen dies naturally at a rate of $\mu_{T_{b}}>0$. The macrophages natural death rate is represented by $\mu_{m}$. Finally, the HIV-1 cytotoxic T-cells themselves are produced from the source $s_{c}$ of HIV specific CTLs [28] [29]. The source represent the new CD8 ${ }^{+}$T-cells from the thymus and those from the precursors. The naive $\mathrm{CD}^{+} \mathrm{T}$-cells are then differentiated into HIV specific CTLs which can also proliferate at rate $\rho_{T}$ due to infection by the HIV. A process dependent on the help by the $\mathrm{CD} 4^{+} \mathrm{T}$-cells and antigen present from the B-cells [28] [29]. The CTLs can also die naturaly at a rate $\mu_{c}$. Thus these immune dynamics facts can be represented by the following system of equations:

$$
\begin{aligned}
\dot{T}(t)= & s_{T}-\mu_{T} T(t)+r_{t}\left(\frac{V(t)}{V(t)+D_{v}}\right) T(t)+r_{m}\left(\frac{T_{b}(t)}{T_{b}(t)+D_{T_{b}}}\right) T(t) \\
& -\left(\frac{k_{v} T(t)}{T(t)+B_{T}}\right) V(t)-\left(\frac{k_{1} V(t)}{1+a_{0} C(t)}\right) T(t), \\
\dot{T}^{*}(t)= & \left(\frac{k_{1} V(t)}{1+a_{0} C(t)}\right) T(t)-h C(t) T^{*}(t)-\alpha T^{*}(t), \\
\dot{V}(t)= & \frac{N_{T} \alpha T^{*}(t)}{1+b_{0} C(t)}(t)+\frac{N_{m} \alpha_{m} k_{2} M(t) V(t)}{1+b_{0} C(t)}-\mu_{v} V(t), \\
\dot{M}(t)= & \mu_{M}\left(M_{0}-M(t)\right)-k_{2} M(t) V(t)+r_{2 m} M(t) V(t)+r_{1 m} M(t) T_{b}(t), \\
\dot{T_{b}}(t)= & r_{T_{b}} T_{b}(t)\left(K-T_{b}(t)\right)-\mu_{T_{b}} T_{b}(t)-T_{b}(t)\left(k_{3} T(t)+k_{4} M(t)\right), \\
\dot{C}(t)= & s_{c}+\rho_{T} C(t) T(t) V(t)-\mu_{c} C(t) .
\end{aligned}
$$

\section{Initial Conditions and Reproductive Number, $\boldsymbol{R}_{0}$}

\subsection{Disease Free State $\left(E_{0}\right)$}

The disease free equilibrium is given by $E_{0}$ where the abundance of the uninfected $\mathrm{CD}^{+} \mathrm{T}$-cells, infected CD4 ${ }^{+} \mathrm{T}$-cells, HIV, macrophages, Mtb, and CTL T-cells are given by $T_{c}, T_{c}^{*}, V_{c}, M_{c}, T_{b c}, C_{c}$, respectively. This is found when all pathogen populations are zero. It is given by:

$$
E_{0}=\left(T_{c}, T_{c}^{*}, V_{c}, M_{c}, T_{b c}, C_{c}\right)=\left(\frac{s_{T}}{\mu_{T}}, 0,0, M_{0}, 0, \frac{s_{c}}{\mu_{c}}\right)
$$

\subsection{Reproductive Number, $\boldsymbol{R}_{0}$}

The basic reproductive number $\left(R_{0}\right)$ measures the number of new secondary infections generated by a single co-infected individual cell in the presence of CTLs mechanisms. It 
is a dimensionless parameter which can be used to investigate the potency of the immune system. It can be used to control infections and disease progression so that they are kept at low levels. $R_{0}$ is here given as the dominant eigenvalue of the next generation matrix of the Jacobian matrix by Watmough's method [30]. There are several $R_{0}^{\prime} s$ for system (2), which can be denoted as follows:

- $R_{0 v}$ denoting the reproductive ratio given only when there is only viral infection. This can be defined as the average number of viruses produced by one virus in a mostly susceptible population of $\mathrm{CD} 4^{+} \mathrm{T}$-cells.

- $R_{0 b}$ denoting the reproductive ratio given only when there is $\mathrm{Mtb}$ infection. It is defined as the average number macrophages that can be infected from a single Mtb bacteruim in a mostly susceptible population of macrophages.

- $R_{0}$ denoting the reproductive ratio when there is co-infection and given as

$$
\begin{aligned}
R_{0} & =\left(\frac{\mu_{c}\left[N_{T} \alpha k_{1} s_{T}+\left(\mu_{c}+a_{0} s_{c}\right)\left(\alpha s_{c}+h \mu_{c}\right) N_{m} \alpha_{m} k_{2} M_{0}\right]}{\mu_{T} \mu_{v}\left(\alpha s_{c}+h \mu_{c}\right)\left(\mu_{c}+a_{0} s_{c}\right)\left(\mu_{c}+b_{0} s_{c}\right)}, \frac{\mu_{T} k_{4} M_{0}}{\mu_{T_{b}}+k_{3} s_{T}+K r_{T_{b}}}\right), \\
& =\left(R_{o v}, R_{o b}\right) .
\end{aligned}
$$

Diseases are not competing, therefore an individual can die of either HIV or Mtb or both and so they both contribute to overall $R_{0}$ rather than considering the maximum value of the next generation matrix.

\section{Endemic States Analysis}

The model system (2) has three endemic states: the virally infected state (where only the HIV pathogen is present), the Mtb state (which represents the presence of the Mtb pathogen only) and the co-infected state (where both pathogens are present). The stability analysis of these states is here discussed and analysed using the Jacobian method.

\subsection{Virally Infected State $\left(W_{1}\right)$}

The population density of the uninfected $\mathrm{CD}^{+} \mathrm{T}$-cells, infected $\mathrm{CD} 4^{+} \mathrm{T}$-cells, Virus, Mtb, Macrophages and the HIV specific CTLs at the virally infected endemic state are respectively given by $W_{1}$ as:

$$
W_{1}=\left(T_{1}, T_{1}^{*}, V_{1}, M_{1}, T_{b 1}, C_{1}\right),
$$

where $T_{b 1}=0$. The uninfected $\mathrm{CD} 4^{+} \mathrm{T}$-cells population density is given by

$$
\begin{aligned}
T_{1} & =\frac{\left(1+a_{0} C_{1}\right)\left(h C_{1}+\alpha\right)\left[\mu_{v}\left(1+b_{0} C_{1}\right)-N_{m} \alpha_{m} k_{2} M_{1}\right]}{N_{T} \alpha k_{1}}, \\
& =\frac{T_{c}}{R_{0 v}} .
\end{aligned}
$$

$T_{1}$ is positive when $\mu_{v}\left(1+b_{0} C_{1}\right)>N_{m} \alpha_{m} k_{2} M_{1}$. That is, the cells only exist when the condition is satisfied. The population increases with increase in the inhibition of viral entry, replication rate and direct killings by the CTLs attribute which again shows a 
protective effect by the CTLs. The population of uninfected $\mathrm{CD} 4^{+} \mathrm{T}$-cells decreases with increase in the HIV infection rate and increase in viral production (due to replication and bursting) as explained in system (2), which attributes to increase in $R_{0 v}$. Thus increase in $R_{0 v}$ reduces $T_{1}$ population and vice versa.

The abundance of the infected $\mathrm{CD} 4^{+} \mathrm{T}$-cells at the virally infected state is given by

$$
T_{1}^{*}=\frac{k_{1} V_{1} T_{1}}{\left(1+a_{0} C_{1}\right)\left(h C_{1}+\alpha\right)} .
$$

This is effectively reduced by increase in the inhibition of viral infection and direct killing of the virus by the CTLs. The population however, increases with increase in the viral population which inturn increases the reproductive ratio.

The viral load at this endemic steady state is given by the expression:

$$
V_{1}=\frac{N_{T} \alpha T_{1}^{*}}{\mu_{v}\left(1+b_{0} C_{1}\right)-N_{m} \alpha_{m} k_{2} M_{1}} .
$$

The equilibrium value of the viral load is dependent on the average number of the new viruses produced by the infected macrophages as well as infected $\mathrm{CD} 4^{+} \mathrm{T}$-cells. Increase in the viral production attribute to an increase in the viral load. This viral population is however, reduced by the effectiveness of the CTLs to inhibit viral production and replication, a mechanism of the CTLs that reduces viral multiplication and protects the $\mathrm{CD}^{+} \mathrm{T}$-cells. Thus, inclusion of these mechanisms in the new model helps to understand how the virus can be reduced. Positivity of the viral population is possible when $\mu_{v}\left(1+b_{0} C_{1}\right)>N_{m} \alpha_{m} k_{2} M_{1}$.

The macrophage population is given by

$$
M_{1}=\frac{\mu_{M} M_{0}}{\mu_{M}+V_{1}\left(k_{2}-r_{2 m}\right)} .
$$

This population is sustained when $\mu_{M}>V_{1}\left(r_{2 m}-k_{2}\right)$ and it is reduced with increase in the viral load.

Finally, the HIV specific CTLs population density is given by:

$$
C_{1}=\frac{s_{c} T_{c} R_{0 v}}{\mu_{c} T_{c} R_{0 v}-\rho_{T} V_{1}}
$$

Increase in the viral population density increases the $R_{0 V}$ which then stimulate more CTL to be released to either directly kill the virus via their phagocytic nature or inhibit replication and more infections although it also depends on the rate of production of the CTLs from the source. This is possible when the condition $R_{0 v}>\frac{\rho_{T} V_{1}}{\mu_{c} T_{c}}$ is satisfied.

\subsection{Mtb Equilibrium State $\left(W_{2}\right)$}

The second endemic equilibrium state is the Mtb infected steady state where the viral load and the infected T-cell populations are all zeros and is given by:

$$
W_{2}=\left(T_{2}, T_{2}^{*}, V_{2}, M_{2}, T_{b 2}, C_{2}\right) \text {, }
$$


where $T_{2}^{*}=V_{2}=0$ and $T_{2}, M_{2}, T_{b 2}, C_{2}$ are the equilibrium values showing the abundance of the uninfected T-cells, macrophages, Mtb population and the HIV specific CTLs, respectively, and the values are given by expressions (12)-(15). The infected $\mathrm{T}$-cell equilibrium value at the Mtb endemic steady state is given by:

$$
T_{2}=\frac{s_{T}}{\mu_{T}-r_{m}\left(\frac{T_{b 2}}{T_{b 2}+D_{T_{b}}}\right)} .
$$

The uninfected CD4 $4^{+} \mathrm{T}$-cells density exist only if $\mu_{T}\left(T_{b 2}+D_{T_{b}}\right)>r_{m} T_{b 2}$. It depends mainly on the rate at which they are being produced, their natural death and the proliferation rate due to infection by the Mtb. More Mtb infections trigger release of more $\mathrm{CD} 4^{+} \mathrm{T}$-cells. The protective effect of the CTLs is not exihited at this equilibrium state since they are HIV specific.

The macrophages are given by:

$$
M_{2}=\frac{\mu_{M} M_{0}}{\mu_{M}-r_{1 m} T_{b 2}} .
$$

This value is dependent on the interactions of the macrophages with the Mtb pathogen and the natural death rate for the macrophages. When Mtb approaches zero then the macrophage population goes to the uninfected steady state value of $M_{0}$. The value of the CTLs at this endemic state is the same as the one at the uninfected steady state since the CTLs are not involved in the controlling of Mtb as they are HIV specific. Thus

$$
C_{2}=C_{c}=\frac{s_{c}}{\mu_{c}} .
$$

The Mtb population at the Mtb endemic steady state is given by the following expression:

$$
T_{b 2}=\frac{K r_{T_{b}}-\mu_{T_{b}}-k_{3} T_{2}-k_{4} M_{2}}{r_{T_{b}}} .
$$

The equilibrium value depends totally on the interaction of the Mtb with the macrophages and the killing by the $\mathrm{CD}^{+} \mathrm{T}$-cells together with the Mtb natural death rate and the Mtb carrying capacity. Mtb exists only if the following condition is satisfied

$$
K>\frac{\mu_{T_{b}}+k_{3} T+k_{4} M_{2}}{r_{T_{b}}} .
$$

\subsection{Co-Infected State}

The last endemic state is the co-infected endemic state where both pathogens are present given by

$$
W_{3}=\left(T_{3}, T_{3}^{*}, V_{3}, M_{3}, T_{b 3}, C_{3}\right),
$$

where $T_{3}, T_{3}^{*}, V_{3}, M_{3}, T_{b 3}, C_{3}$ are the equilibrium values at the co-infected steady state. The abundance of the viral load and the CTL equilibrium values are given by (8) 
and (14) respectively, same values as the ones at the virally infected steady state except that the uninfected T-cell values are replaced by $T_{3}$. The Mtb population again is still the same as the one at the Mtb endemic state, expression (15). The abundance of the uninfected $\mathrm{CD} 4^{+} \mathrm{T}$-cells at the co-infected steady state is given by

$$
\begin{aligned}
T_{3} & =K\left(R_{0 b}+1\right)+Q-B_{T}(A+B) \\
& \pm \sqrt{\left(K\left(R_{0 b}+1\right)+Q-B_{T}(A+B)\right)^{2}+4(A+B) B_{T} Q},
\end{aligned}
$$

where $Q, A, B$ are given by equations in Appendix (10.1). This shows that the uninfected $\mathrm{CD}_{4}{ }^{+} \mathrm{T}$-cells are reduced more at the co-infected steady state due to the virus and also due to the Mtb infection. The macrophage density at the co-infected steady state is given by

$$
M_{3}=\frac{\mu_{M} M_{0}}{\mu_{M}+V\left(k_{2}-r_{2 m}\right)-r_{1 m} T_{b}} .
$$

where $\mu_{M}>V_{3}\left(r_{2 m}-k_{2}\right)+r_{1 m} T_{b}$ for the macrophages to be feasible at the co-infected equilibrium state. Thus, the value of the macrophages are seriously reduced in a co-infected individual due to the presence of both pathogens which reduce the macrophage population. The uninfected T-cells steady state value for the co-infected endemic state cannot be explicitly found since it involves a polynomial of higher order. However, it can be noted that the abundance of uninfected $\mathrm{CD} 4^{+} \mathrm{T}$-cells is reduced due to the presence of both pathogens. This is only possible when we have both the $R_{0 v}$ and the $R_{0 b}$ being greater than unity for the co-infected state to be established.

\section{Stability Analysis}

\subsection{Disease Free State, $E_{0}$}

Stability analysis of all the steady states are here done using the characteristic polynomial of the Jacobian matrix at each equilibrium state for all our equilibrium states. The stability analysis of the uninfected steady is analysed using $J_{1}$ in Appendix (10.2). The eigenvalues are calculated by solving the characteristic polynomial

$$
\left(\lambda-\mu_{T}\right)\left(\lambda-\mu_{M}\right)\left(\lambda-\mu_{c}\right)\left(\lambda-b_{2}\right)|(\lambda-a)(\lambda-d)-b c|=0,
$$

where $a, b, b_{2}, c$, and $d$ are given in Appendix (10.2). All the four eigenvalues are negative when

$$
K<\frac{\mu_{T_{b}}+k_{3} s_{T}+\mu k_{4} M_{0}}{r_{T_{b}}}=K^{*} .
$$

The other two eigenvalues are obtained from the quadratic equation $((a-\lambda)(d-\lambda)-b c)$ which has negative roots if and only if $1-(a+d)<0$ and $a d-b c>0$. This is only possible if

$$
\begin{aligned}
N_{T} & <\frac{\mu_{T}\left(\mu_{c}+a_{0} s_{c}\right)\left(\mu_{c}+b_{0} s_{c}\right)\left(-h s_{c}-\alpha \mu_{c}\right)\left(\mu_{v}-\alpha_{m} N_{m} k_{2} M_{0}\right)}{\alpha k_{1} s_{T} \mu_{c}} \\
& =N^{*} .
\end{aligned}
$$


Stability of $E_{0}$ requires that the Mtb carrying capacity $K<K^{*}$ is satisfied by condition (20). The same condition is required for system (1) that $K<K_{c}$ [22], where $K_{c}=K^{*}$ are the critical values for the Mtb carrying capacity in system (1) and (2), respectively. This is due to the fact that the CTLs incorporated in system (2) are only HIV specific and therefore does not change mechanisms in the Mtb dynamics. Thus with or without the CTL factors, stability of the disease free equilibrium is achievable when $K<K^{*}=K_{c}$. Condition given by 18 is true when $R_{0 b}<1$. The second condition for stability of $E_{0}$ is dependent on the number of produced by infected CD $4^{+}$ T-cells, $N_{T}$. Stability depends on $N^{*}$ which is dependent on the CTL's ability to directly kill the virus, inhibit viral infection and replication over and above the same other factors as system (1). Also of interest is the reduction by the CTL factor to production of more viruses by macrophages $\alpha_{m} N_{m}$. Therefore $N^{*} \rightarrow 0$ as either one or a combination of $\left(a_{0}, b_{0}, h\right) \rightarrow \infty$. Condition $(21)$ is only possible when $R_{0 v}<1$. Thus $E_{0}$ is stable when both $R_{0 v}, R_{0 b}$ are less than unity. Unlike in system (1), where stability of uninfected state depends solely on the $\mathrm{CD} 4^{+} \mathrm{T}$-cells production and macrophages ability to produce more viruses only as shown by $N_{c}$ [22], in system (2) stability of $E_{0}$ happens o when $N_{T}<N^{*}\left(R_{0 v}<1\right)$, and $N^{*}$ is reduced as we increase CTL mechanisms. This limits disease progression, suppose we are to increase these mechanisms since $R_{0 v}$ is reduced., new/secondary infections by the virus is reduced. Therefore increasing these CTL mechanisms results in reducing the overal $R_{0}$, which also have an impact of protecting the $\mathrm{CD} 4^{+} \mathrm{T}$-cells since new infections are reduced. If a vaccine can be improvised, it will be helpful to enhance these CTLs factors to reduces viral replication and new infections. $E_{0}$ loose its stability to either $W_{1}, W_{3}$ or $W_{2}$ through the critical values of $K^{*}$ and $N^{*}$.

\subsection{Virally Infected State}

Stability of $W_{1}$ cannot be done explicitly from the eigenvalues of $J_{2}$ in appendix (10.3) since the steady state values for the viral load are not explicitly found. However, one of the eigenvalues, can be easily read from $J_{2}$ and is equal to one of the eigenvalues from $J_{1}$ in (10.2) corresponding to condition (20). This implies that for the virally infected state to be stable we need to have $R_{0 b}<1$ same condition established by Kirshner [22] for system (1). When $R_{0 b}>1$ then we have the virally endemic steady state loosing stability to either $W_{2}$ or $W_{3}$. This leads to a state where by the virus establishes itself fast and hence quick progression to AIDS. It is therefore necessary to keep $R_{0 b}<1$ so that the clinical picture of the HIV infection is maintained at low levels by the immune system. Any changes in the Mtb carrying capacity $K^{*}$ have a profound effect on the stability of the Stability of $W_{1}$. Thus, disturbances in the Mtb natural death rate and Mtb interaction with both the T-cells and the macrophages then brings qualitative changes to the stability of the virally infected steady state and hence the clinical picture of HIV infected individual in particular. Therefore $K^{*}$ can also be used to control infection. 


\subsection{Mtb Infectected State}

At Mtb infected state stability is characterised by the characteristic polynomial of $\left|J_{3}-\lambda I\right|=0$ given by

$$
\left|\begin{array}{cc}
c-\lambda & f \\
d & g-\lambda
\end{array}\right|[(a-\lambda)(m-\lambda)(c-\lambda)-j(a-\lambda)(i-\lambda)+b(m-\lambda)-b j]=0
$$

where $J_{3}, a, b, c, d, f, g, h, i, j, k, m, n, p$ are all given in Appendix (10.4). One of the eigenvalues can be read directly from the Jacobian that is $\lambda_{1}=-\mu_{c}$ the other two eigenvalues are calculated from the characteristic polynomial of the following determinant

$$
\left|\begin{array}{cc}
c-\lambda & f \\
d & g-\lambda
\end{array}\right|=0
$$

The roots are negative if and only if $-d f+c g>0$ which implies giving the same condition as in requirement condition (21). This condition is true when $N_{T}<N^{*}$ that is $\left(R_{0 v}<1\right)$ and when $T=T_{c}$ and $M=M_{c}$ given by Equation (4). Stability of the Mtb state state arises when the reproductive ratio for the HIV is kept below unity. However, it must also be noted that $R_{0 v}$ mainly depends on the effector mechanisms of the CTLs such that any slight changes in these CTL mechanisms significantly changes condition (21) hence the stability of the Mtb infected endemic steady state. The state of the last three eigenvalues can be determined from the following theorem.

Theorem 1 Let $P_{H}(s)=b_{0}+b_{1} s+b_{2} s^{2}+b_{3} s^{3}+\cdots+b_{n} s^{n}$ with $b_{i}$ real and $b_{0} \geq 0$ which is assumed if $b_{0}<0$ then scale the whole expression with -1 which does not change the zeros of $P_{H}$. Then $P_{H}$ have roots with negative real parts if and only if the routh determinants of the polynomial $\Delta_{i}=b_{1}, \Delta_{2}, \cdots, \Delta_{n}$ are all positive where

$$
\Delta_{i}=\left|\begin{array}{ccccc}
b_{1} & b_{3} & - & - & b_{2 i-1} \\
b_{0} & b_{2} & - & - & b_{2 i-2} \\
0 & b_{1} & b_{3} & - & b_{2 i-3} \\
- & b_{0} & b_{2} & - & b_{2 i-4} \\
- & - & - & - & b_{i}
\end{array}\right|
$$

Thus, from the theorem we have the Mtb infected endemic state stable since the other roots of the Jacobian matrix have negative real parts since the routh determinants of the polynomial $P_{3}(\lambda)=b_{0}+b_{1} \lambda+b_{2} \lambda^{2}+b_{3} \lambda^{3}$ are all positive where

$$
\begin{aligned}
& b_{3}=1>0, \\
& \Delta_{1}=b_{1}=(a m+a c+c m-a j-i j+b)>0, \\
& \Delta_{2}=\left(b_{1} b_{2}-b_{0} b_{3}\right)>0, \\
& b_{0}=(b m-b j+a c m-a i j), \\
& b_{2}=(j-a-m-c) .
\end{aligned}
$$

The Mtb infected steady state can only be stable also when the $T_{b}>0$ which is given by the condition 


$$
K>\frac{\mu_{T_{b}}+k_{3} T+k_{4} M}{r_{T_{b}}} .
$$

Therefore, stability of the Mtb infected steady state is stable when $R_{0 b}>1$ and $R_{0 v}<1$. The same critical points $K^{*}$ and $N^{*}$ determine stability for both the Mtb infected and the virally infected endemic states. Thus, there exists a switching behavior on stability between $W_{1}$ and $W_{2}$ via these parameters. $W_{1}$ looses its stability via $K^{*}$ and to $W_{2}$ and also $W_{2}$ to $W_{1}$ via both $K^{*}$ and $N^{*}$. This implies transcritical bifurcations at these points although they are not analysed in detail in this paper.

\subsection{Co-Infected State}

The co-infected endemic steady state can only be stable when the number of pathogens for both diseases are greater than zero and this corresponds to the condition when $N_{T}>N^{*}$ and when $K>K^{*}$. Thus both $R_{0 v}$ and $R_{0 b}$ are greater than unity, implying that as long as both pathogens exist, an individual always remains in the co-infected state which makes it very difficult to control the disease.

\section{Numerical Simulations}

Matlab 6.5 version was used for all our simulations for both models using ODE45 solver. Simulations on this model give us a portrait of the general behaviour of immune cells population in the presence of the HIV and Mtb pathogens. We are also concerned about the parameters which are of importance in stabilising the model and the ranges in which the system is stable and unstable. Table 1 shows all the parameter values used for all our simulations are here in used. The initial condition for systems (1) and (2) are given as $T(0)=1500 \mathrm{~mm}^{-3}, T^{*}(0)=0.01 \mathrm{~mm}^{-3}, \quad V(0)=0.001 \mathrm{~mm}^{-3}, \quad M(0)=$ $100 \mathrm{~mm}^{-3}, T_{b}(0)=0.001 \mathrm{~mm}^{-3}$ and $C(0)=250 \mathrm{~mm}^{-3}$ adopted from [22] [27].

\section{Effects of CTL Mechanisms}

\subsection{Effects of CTLS on $R_{0}$}

The presence of the lytic factor is almost insignificant in a co-infected state since it depicts the severe stage of HIV and Mtb. The different mechanisms affect the reproductive ratio differently. Figure 1(a) shows how $R_{e v}$ changes when there is only inhibition of infection, replication and direct killing by CTLs, given by $R_{a}, R_{b}$, and $R_{h}$, respectively. The ratios generally decrease with CTL mechanisms. It shows that the lytic factors are more essential than the non-lytic factors in the order of $h, a_{0}$ and $b_{0}$. It also shows that although the reproductive ratio is reduced with increase in CTL mechanisms, it will never be reduced to zero. This is because of the nature of HIV which is a retrovirus thus we will always have the virus once a person is infected. A combination of the different mechanisms here not shown was also done numerically and observed that the reproductive ratio is reduced more when all the mechanisms are enhanced [31]. Removing any one of the CTL mechanisms will result in a high ratio which depends on $N_{T}$ and $K$. Thus condition $h C \gg \alpha$ means the rate of CTL 
Table 1. Numerical values for the simulations.

\begin{tabular}{|c|c|c|c|c|}
\hline Parameter & Symbol & Value & Units & Ref. \\
\hline Rate $\mathrm{CD} 4^{+} \mathrm{T}$-cells becomes infected by free virus & $k_{1}$ & $2.4 \times 10^{-5}$ & $\mathrm{~mm}^{-3} \cdot \mathrm{d}^{-1}$ & {$[22]$} \\
\hline Rate of macrophage infection by HIV & $k_{2}$ & $2 \times 10^{-6}$ & $\mathrm{~mm}^{-3} \cdot \mathrm{d}^{-1}$ & [22] \\
\hline Rate at which uninfected T-cells kill Mtb & $k_{3}$ & 0.5 & $\mathrm{~mm}^{-3} \cdot \mathrm{d}^{-1}$ & {$[22]$} \\
\hline Rate at which macrophages kill Mtb & $k_{4}$ & 0.5 & $\mathrm{~mm}^{-3} \cdot \mathrm{d}^{-1}$ & {$[22]$} \\
\hline HIV apoptosis rate & $k_{v}$ & $2 \times 10^{-3}$ & $\mathrm{~mm}^{-3} \cdot \mathrm{d}^{-1}$ & [27] \\
\hline Cytotoxic T cell death & $\mu_{c}$ & 1.5 & $\mathrm{~d}^{-1}$ & [27] \\
\hline Rate new CTLs are produced & $s_{c}$ & 10 & $\mathrm{~mm}^{-3} \cdot \mathrm{d}^{-1}$ & [27] \\
\hline Production of T-cells & $S_{T}$ & 20 & $\mathrm{~mm}^{3} \mathrm{~d}$ & {$[22]$} \\
\hline Death rate of uninfected $\mathrm{T}$ cell population & $\mu_{T}$ & 0.02 & $\mathrm{~d}^{-1}$ & {$[22]$} \\
\hline Macrophage death rate & $\mu_{M}$ & 0.03 & $\mathrm{~d}^{-1}$ & {$[22]$} \\
\hline Natural death of HIV & $\mu_{v}$ & 2.4 & $\mathrm{~d}^{-1}$ & [22] \\
\hline Natural death of Mtb & $\mu_{T_{b}}$ & 0.5 & $\mathrm{~d}^{-1}$ & {$[22]$} \\
\hline Stimulation constant & $B_{T}$ & 350 & $\mathrm{~mm}^{-3}$ & [27] \\
\hline Virus cytopathic rate & $\alpha$ & 0.25 & $\mathrm{~d}^{-1}$ & {$[27]$} \\
\hline Rate of viral production by HIV burst & $\alpha_{m}$ & 0.25 & $\mathrm{~d}^{-1}$ & {$[27]$} \\
\hline CTL proliferation rate & $\rho_{T}$ & $1 \times 10^{-5}$ & $\mathrm{~mm}^{-3} \cdot \mathrm{d}^{-1}$ & {$[27]$} \\
\hline Proliferation of uninfected $\mathrm{T}$ cell by virus & $r_{T}$ & 0.02 & $\mathrm{~d}^{-1}$ & {$[22]$} \\
\hline Proliferation of uninfected T cell due to Mtb infection & $r_{m}$ & 0.01 & $\mathrm{~d}^{-1}$ & est \\
\hline Maximal proliferation of the $M$ tuberculosis population & $r_{T_{b}}$ & 1.0 & $\mathrm{~d}^{-1}$ & {$[22]$} \\
\hline HIV infection reduction rate by CTLs & $a_{0}$ & 0.025 & $\mathrm{~mm}^{3}$ & [27] \\
\hline HIV replication reduction rate by the CTLs & $b_{0}$ & 0.05 & $\mathrm{~mm}^{3}$ & [27] \\
\hline Rate of HIV infected are killed by CTLs & $h$ & $2 \times 10^{-3}$ & $\mathrm{~mm}^{-3} \cdot \mathrm{d}^{-1}$ & {$[27]$} \\
\hline Number of viruses produced by infected T-cells & $N_{T}$ & 1000 & $\mathrm{~mm}^{-3}$ & {$[22]$} \\
\hline Number of viruses produced by infected macrophages & $N_{m}$ & 500 & $\mathrm{~mm}^{-3}$ & [27] \\
\hline Stimulation constant for the HIV & $D_{v}$ & 400 & $\mathrm{~mm}^{-3}$ & est \\
\hline Stimulation constant for the Mtb & $D_{T_{b}}$ & 450 & $\mathrm{~mm}^{-3}$ & {$[22]$} \\
\hline Mtb carrying capacity & $K$ & 800 & $\mathrm{~mm}^{-3}$ & {$[22]$} \\
\hline Equilibrium value for the macrophage population & $M_{0}$ & 100 & $\mathrm{~mm}^{-3}$ & {$[22]$} \\
\hline
\end{tabular}

mediated killings is low relative to the rate of cell-mediated killings and $\alpha \gg h C$ means that the rate of virus induced $\mathrm{CD} 4^{+} \mathrm{T}$-cells killings is very high relative to the rate of CTL mediated killing [27]. The reproductive ratio here shows that for both high and low values of CTLs ( $C \approx \infty$ and $C \approx 0$ ) and $h C \ll \alpha$, then the order of the ratios is $R_{01}>R_{03}>R_{04}>R_{02}$ [31]. An indication that the non-lytic arm of the CTLs is more essential than the lytic arm when $h C \gg \alpha$ for both low and high levels of the CTLs, since the ratios are generally low when non-lytic arm are in operation than when the 


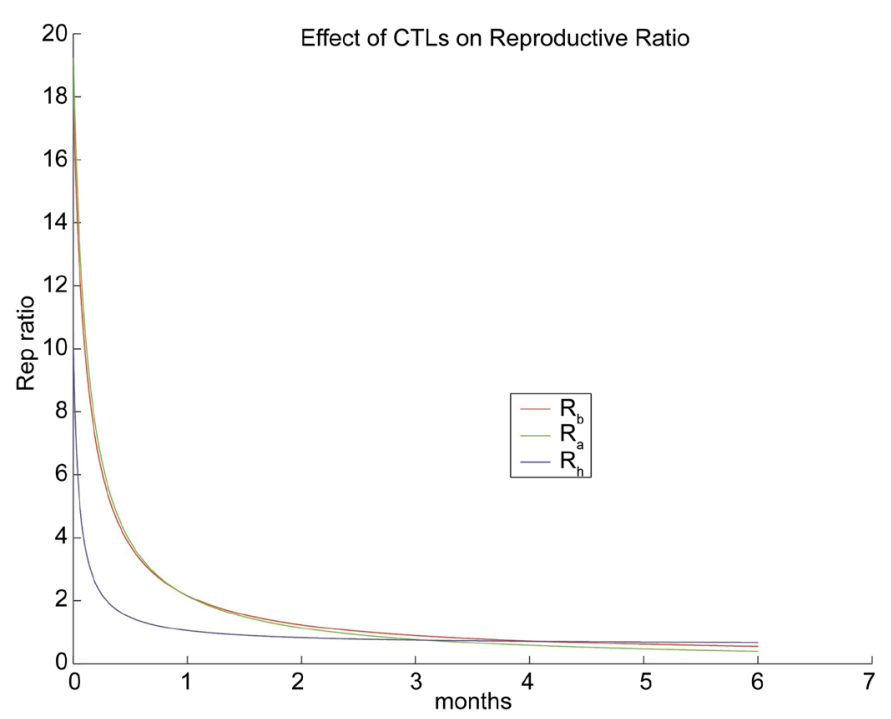

(a)

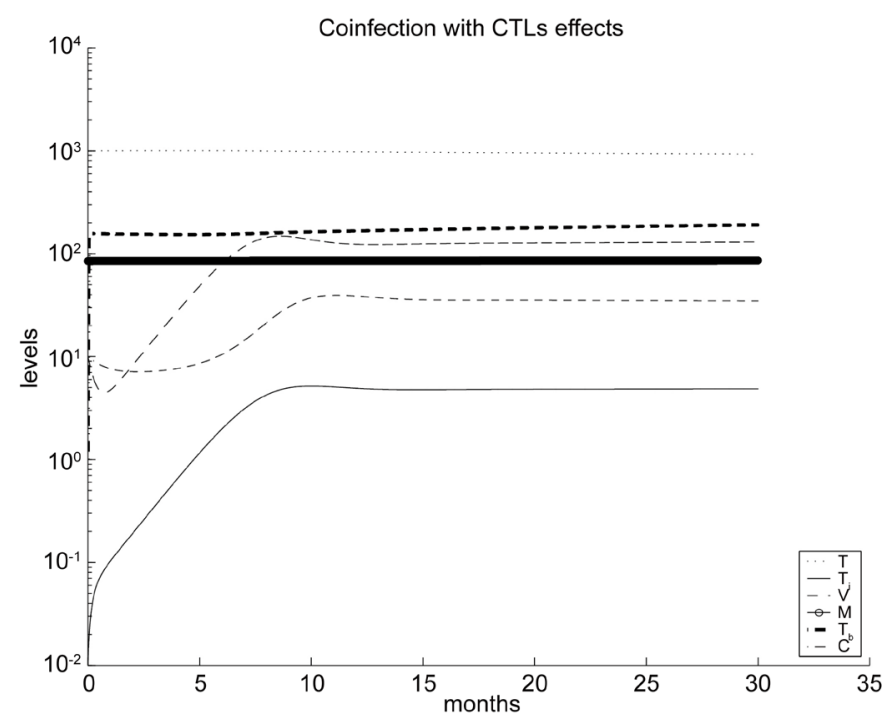

(c)

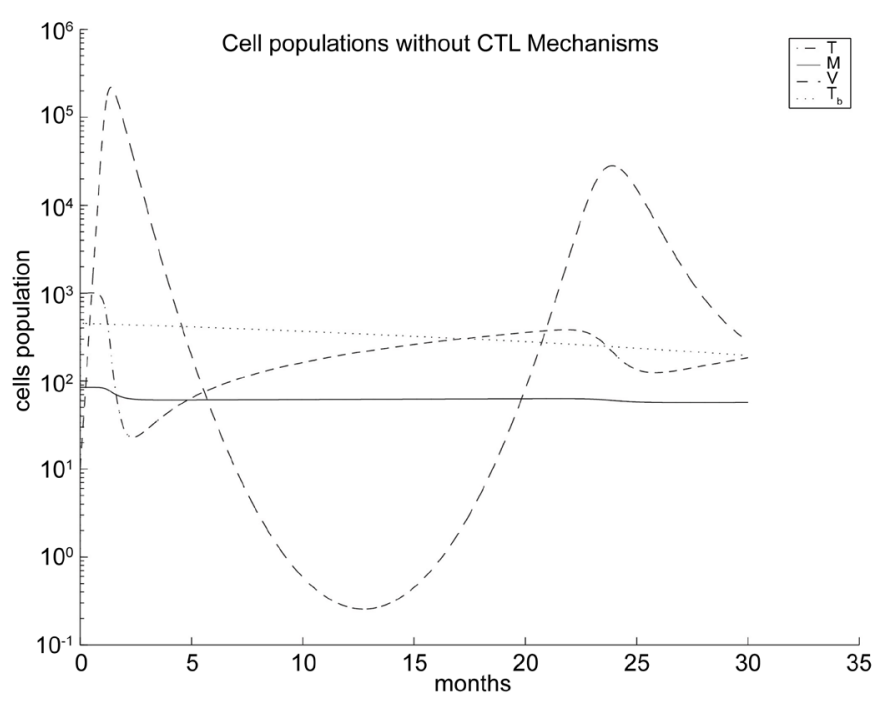

(b)

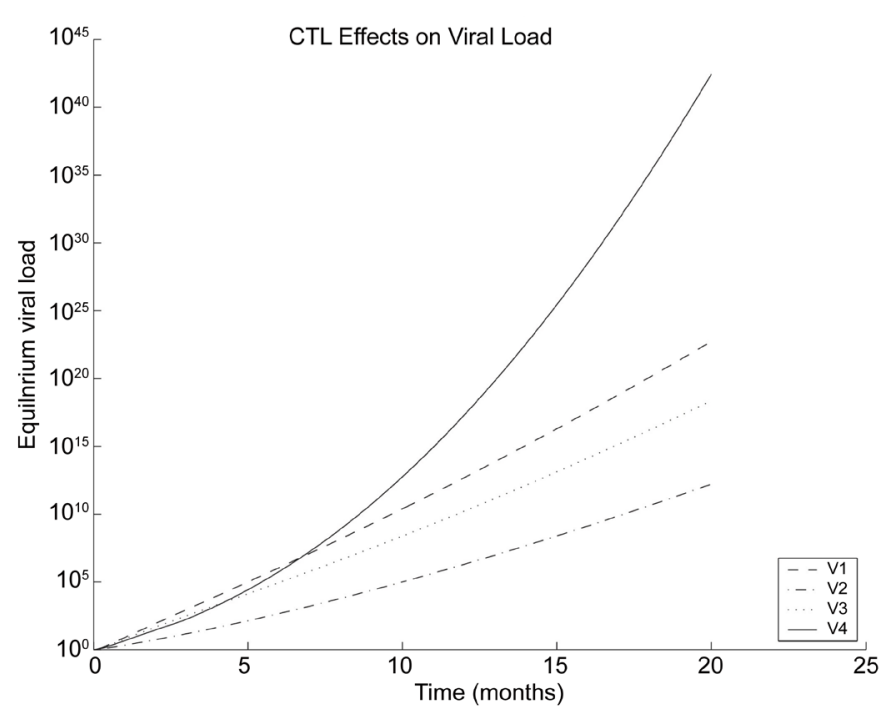

(d)

Figure 1. Effects of different CTL mechanisms on the reproductive ratio and Uninfected CD4 ${ }^{+} \mathrm{T}$-cells. (a) Graph of reproductive ratios, (b) Cell populations propagations without CTLs, (c) Cell populations propagations with CTLs mechanisms incorporated, (d) Effects of CTLs on viral load.

lytic arm is in operation. Here the $R_{0 i}, i=1: 4$ are defined in terms of $i$ given by system (23). Hence, chances of controlling infection by the immune system are increased when the non-lytic factors are in operation. If $h C \gg \alpha$, then the order of the reproductive ratios is given by $R_{02}>R_{01}>R_{04}>R_{03}$ for both low and high levels of the CTLs. This implies that for this condition, we have the lytic factors being more effective than the non- lytic factors as ratios are lower when the lytic factors are in operation than when only non lytic factors is involved. In general, we also have lower values of the reproductive ratios for higher values of CTLs being lower than for low CTL values indicating the importance of increased involvement of the effector mechanisms of the 
CTLs. Therefore the CTLs have a very vital role to play in the control of HIV infection in a co-infected individual as it lowers significantly the reproductive ratio hence protect $\mathrm{CD}^{+} \mathrm{T}$ cell infection by keeping a low viral load and hence keeping the Mtb carrying capacity lower than $K^{*}$.

\subsection{Cell Population}

There is also evidence of the uninfected $\mathrm{CD} 4^{+} \mathrm{T}$-cell depletion due the presence of CTL mechanisms as shown by Figure 1(b) and Figure 1(c). The viral population can never reach out to higher levels above $10^{2}$ in the presence of CTLs as shown in Figure 1(c) but reach up to levels of $10^{4}$ in the model by Kirshner [22] as in Figure 1(b). Thus the presence of these mechanisms limits the viral population growth and hence limit the depletion of $\mathrm{CD} 4^{+} \mathrm{T}$-cells. This has an effect of reducing the rate of progression to the AIDS state. The different combinations of the CTL mechanisms can be analysed on the equilibrium values of all the variables. Each of the equilibrium values is then plotted against time for the different combinations. In this paper, only the different combinations under viral population is analysed although all the other populations were done but not represented as in Figure 1(d). It must also be noted that the other results agree with those on the viral load. Figure 1(d) shows that a combination of direct killing of the virus and hinderance of viral replication will go a long way in reducing the viral load. $V_{i}^{\prime} S$ represent the following scenarios:

$$
\begin{aligned}
& i=1, \text { represents } a_{0}=b_{0}, h \neq 0 \\
& i=2, \text { represents } a_{0} \neq 0, b_{0} \neq 0, h=0 \\
& i=3 \text {, represents } a_{0} \neq 0 \neq, b_{0}=0, h \neq 0 \\
& i=4, \text { represent } a_{0}=0, b_{0} \neq 0, h \neq 0
\end{aligned}
$$

\section{Conclusions}

The HIV specific CTLs have a very big role to play in limiting viral spread and controlling infection by the virus in the immune system, which means that they have profound effects to the dynamics of the co-infection of Mtb and HIV in general. In the early stages of infection when the HIV specific CTLs are still in small amounts, we have noticed that the lytic factors are more important than the non-lytic factors and reaching the AIDS state is accelerated when the lytic factors are limited in the immune system, a weakness of our basic model by Kirshner [22] since it had no mechanisms of the CTL effects. CTLs are believed to be in abundance at the chronic stage of HIV infection and hence when $C \approx \infty$, the non-lytic factors become more effective than the lytic ones. This implies that both arms of the CTLs are essential for the immune system to control infection. The same conditions were deduced by other authors [27].

The study shows that both the lytic factors and the non-lytic factors are important in the controlling of HIV infection in a co-infected individual. The non-lytic mechanisms are more effective in controlling infection in both cases when rate of CTL mediated killings is low relative to the rate of cell-mediated killings. The lytic factors are more 
important when rate of virus induced $\mathrm{CD} 4^{+} \mathrm{T}$-cells killings is very high relative to the rate of CTL mediated killing regardless of the CTL levels. However, the lytic arm is more important when it comes to control infection than replication since it is believed that CTLs will remain high and non-lytic arms are more important in controlling replication of the virus as they are believed to be more effective at the chronic stage of the infection [27] as also confirmed by Figure 1(a). The CTLs' ability to hinder viral replication protects the clinical picture of a co-infected individual hence protecting the individual from fast AIDS stage as witnessed by Figure 1(a), Figure 1(b). Effectively the presence of CTLs reduces the viral load levels and protects the $\mathrm{CD} 4^{+} \mathrm{T}$ cell levels. Thus, we can conclude that the effector mechanisms of the HIV specific CTLs are relevant in controlling infection by the immune system although there is need to determine which on-lytic factors are more important at the chronic phase. The increase in the CTLs mechanisms reduces viral multiplication, which results on the reduction of viral load, with the non-lytic factors being more effective in viral load reduction. Reduction of the viral load protects the macrophages too, thereby slowing down the rate of progression to AIDS. Thus, if drugs can be put in place to enhance these CTL mechanisms either as a vaccine or treatment, then rate of depletion of CD4 count is reduced and macrophages are protected. Ultimately, the progression to AIDS state is reduced thereby increasing the life span of individual co-infected by HIV and Mtb.

\section{Acknowledgements}

This research was made possible due to the support and insights from Dr G. Magombedze. Thank you very much.

\section{References}

[1] Shankarkumar, U. and Shankarkumar, A. (2011) Role of HLA-A, HLA-B, HLA-DRB1 and HLADQB1 Alleles in HIV-1 Patients with Pulmonary Tuberculosis Co-Infection from Western India. World Journal of AIDS, 1, 136-138. https://doi.org/10.4236/wja.2011.14019

[2] Dye, C., Scheele, S., Dolin, P., Pathania, V. and Raviglione, M.C. (1999) Consensus Statement. Global Burden of Tuberculosis: Estimated Incidence, Prevalence, and Mortality by Country. WHO Global Surveillance and Monitoring Project. JAMA, 282, 677-686. https://doi.org/10.1001/jama.282.7.677

[3] Selwyn, P.A., Hartel, D., Lewis, V.A., Schoenbaum, E.E., Vermund, S.H., et al. (1989) A Prospective Study of the Risk of Tuberculosis among Intravenous Drug Users with Human Immunodeficiency Virus Infection. New England Journal of Medicine, 320, 545-550. https://doi.org/10.1056/NEJM198903023200901

[4] Aaron, L., Saadoun, D., Calatroni, I., Launay O., Memain N., et al. (2004) Tuberculosis in HIV-Infected Patients: A Comprehensive Review. Clinical Microbiology and Infection, 10, 388-398. https://doi.org/10.1111/j.1469-0691.2004.00758.x

[5] Spear, G.T., Kessler, H.A., Rothberg, L., Phair, J. and Landay, A.L. (1990) Decreased Oxidative Burst Activity of Monocytes from Asymptomatic HIV-Infected Individuals. Clinical Immunology and Immunopathology, 54, 184-191. https://doi.org/10.1016/0090-1229(90)90080-A

[6] Wahl, S.M., Allen, J.B., Gartner, S., Orenstein, J.M., Popovic M., et al. (1989) HIV-1 and Its 
Envelope Glycoprotein Down-Regulate Chemotactic Ligand Receptors and Chemotactic Function of Peripheral Blood Monocytes. Journal of Immunology, 142, 3553-3559.

[7] Havlir, D.V. and Barnes, P.F. (1999) Tuberculosis in Patients with Human Immunodeficiency Virus Infection. New England Journal of Medicine, 340, 367-373.

https://doi.org/10.1056/NEJM199902043400507

[8] Patel, N.R., Zhu, J., Tachado, S.D., Zhang, J. and Wan, Z. (2007) HIV Impairs TNF-Alpha Mediated Macrophage Apoptotic Response to Mycobacterium tuberculosis. Journal of Immunology, 179, 6973-6980. https://doi.org/10.4049/jimmunol.179.10.6973

[9] Diedrich, C.R. and Flynn, J.L. (2011) HIV-1/Mycobacterium tuberculosis Co-Infection Immunology: How Does HIV-1 Exacerbate Tuberculosis? Infection and Immunity, 79, 1407-1417. https://doi.org/10.1128/IAI.01126-10

[10] Nakata, K., Rom, W.N., Honda, Y., Condos, R., Kanegasaki, S., et al. (1997) Mycobacterium tuberculosis Enhances Human Immunodeficiency Virus-1 Replication in the Lung. American Journal of Respiratory and Critical Care Medicine, 155, 996-1003. https://doi.org/10.1164/ajrccm.155.3.9117038

[11] Shattock, R.J., Friedland, J.S. and Griffin, G.E. (1993) Modulation of HIV Transcription in and Release from Human Monocytic Cells Following Phagocytosis of Mycobacterium tuberculosis. Research in Virology, 144, 7-12. https://doi.org/10.1016/S0923-2516(06)80005-1

[12] Zhang, Y., Nakata, K., Weiden, M. and Rom, W.N. (1995) Mycobacterium Tuberculosis Enhances Human Immunodeficiency Virus-1 Replication by Transcriptional Activation at the Long Terminal Repeat. Journal of Clinical Investigation, 95, 2324-2331. https://doi.org/10.1172/JCI117924

[13] Orenstein, J.M., Fox, C. and Wahl, S.M. (1997) Macrophages as a Source of HIV during Opportunistic Infections. Science, 276, 1857-1861. https://doi.org/10.1126/science.276.5320.1857

[14] Mancino, G., Placido, R., Bach, S., Mariani, F., Montesano, C., et al. (1997) Infection of Human Monocytes with Mycobacterium Tuberculosis Enhances Human Immunodeficiency Virus Type 1 Replication and Transmission to T-Cells. Journal of Infectious Diseases, 175, 1531-1535. https://doi.org/10.1086/516494

[15] Ignatowicz, L., Mazurek J., Leepiyasakulchai, C., Skold, M., Hinkula, J., et al. (2012) Mycobacterium Tuberculosis Infection Interferes with HIV Vaccination in Mice. PLOS ONE, 7, e41205. https://doi.org/10.1371/journal.pone.0041205

[16] Pawlowski, A., Jansson, M., Skold, M, Rottenberg, M.E. and Kallenius, G. (2012) Tuberculosis and HIV Co-Infection. PLOS Pathogens, 8, e1002464.

https://doi.org/10.1371/journal.ppat.1002464

[17] Khan, F.A., Minion, F., Pai, M., Royce, S., Burman, W., Harries, A.D. and Menzies, D. (2010) Treatment of Active Tuberculosis in HIV-Co-Infected Patients: A Systematic Review and Meta-Analysis. Clinical Infectious Diseases, 50, 1288-1299.

https://doi.org/10.1086/651686

[18] Ramkissoon, S., Mwambi, H.G. and Matthews, A.P. (2012) Modelling HIV and MTB Co-In- fection including Combined Treatment Strategies. PLoS ONE, 7, e49492. https://doi.org/10.1371/journal.pone.0049492

[19] Sharada, R.M., Rani, H.S., Pydi, S.S., Subbanna, J. and Vallur, V.L. (2012) CD38 Expression on CD8+ Cells-Its Influence on Development of Tuberculosis in HIV Positive Individuals. Open Journal of Immunology, 2, 65-71. https://doi.org/10.4236/oji.2012.22008

[20] Ogg, G.S., Dunbar, P.R., Rowland-Jones, S.L., Cerundolo, V. and McMichael, V.J. (1998) Quantitation of HIV-1-Specific Cytotoxic T Lymphocytes and Plasma Load of Viral RNA. 
Science, 279, 2103-2106. https://doi.org/10.1126/science.279.5359.2103

[21] Rowland-Jones, S.L., Dong, T., Fowke, K.R., Kimani, J., et al. (1998) Cytotoxic T Cell Responses to Multiple Conserved HIV Epitopes in HIV-Resistant Prostitutes in Nairobi. Journal of Clinical Investigation, 102, 1758-1765. https://doi.org/10.1172/JCI4314

[22] Kirshner, D. (1999) Dynamics of Co-Infection with M. Tuberculosis and HIV-1. Theoretical Population Biology, 55, 94-109. https://doi.org/10.1006/tpbi.1998.1382

[23] Ganusov, V.V. (2003) The Role of the CTL Response and Virus Cytopathogenecity in the Virus Decline during Antiviral Therapy. Proceedings of the Royal Society of London B, 270, 1513-1518. https://doi.org/10.1098/rspb.2003.2401

[24] Ernst, W.A., Thoma-Uszynski, S., Teitelbaum, R., Ko, C., Hanson, D.A., Clayberger C., Krensky, A.M., Leippe, M., Bloom, B.R., Ganz, T., et al. (2000) Granulysin, a T Cell Product, Kills Bacteria by Altering Membrane Permeability. Journal of Immunology, 165, 71027108. https://doi.org/10.4049/jimmunol.165.12.7102

[25] Klenerman, P., Wu, P. and Phillips, R. (2002) HIV: Current Opinion on Escapology. Current Opinion on Microbiology, 5, 28-36. https://doi.org/10.1016/S1369-5274(02)00339-9

[26] Magombedze, G., Garira, W. and Mwenje, W.E. (2006) Mathematical Models of Chemotherapy of Human Tuberculosis Infection. Biological Sciences, 14, 509-553.

[27] Garira, W., Hove-Musekwa, S.D. and Shiri, T. (2005) Optimal Control of Combined Therapy in a Single Strain HIV-1 Model Electronic. Journal of Differential Equations, 52, 1-22.

[28] Wodarz, D., LLyod, A.L., Jansen, V.A.A. and Nowak, M.A. (1999) Dynamics of Macrophages and T Cell Infection by HIV. Journal of Theoretical Biology, 196, 101-113. https://doi.org/10.1006/jtbi.1998.0816

[29] Wodarz, D. and Nowak, M.A. (2000) Immune Responses and Viral Phenotype:do Replication Rate and Cytopathogenecity Influence Virus load? Journal of Theoratical Medicine, 2, 113-117. https://doi.org/10.1080/10273660008833041

[30] Driessche, V.P. and Watmough, J. (2002) Reproduction Numbers and Sub-Threshold Endemic Equilibria for Compartmental Models of Disease Transmission. Mathematical Biosciences, 180, 29-48. https://doi.org/10.1016/S0025-5564(02)00108-6

[31] Mufudza C. (2008) Analaysing the Effects of Cytotoxic T-Cells in the HIV-1 and MycoBacterium Tuberculosis Co-Infection. Msc Thesis, University of Zimbabwe, Harare, 1-95. 


\section{Appendix}

\subsection{A1}

$$
\begin{aligned}
& Q=s_{T}\left(1+a_{0} C\right)\left[\left(1+a_{0} C\right)\left(1+b_{0} C\right) R_{0 v}+D_{v}\right]\left[K\left(R_{0 b}+1\right)+R_{0 b} D_{T_{b}}\right] \\
& A=\mu_{T}\left(1+a_{0} C\right)\left[\left(1+a_{0} C\right)\left(1+b_{0} C\right) R_{0 v}+D_{v}\right]\left[K\left(R_{0 b}+1\right)+R_{0 b} D_{T_{b}}\right] \\
& B=\left(\left(1+a_{0} C\right)\left(1+a_{0} C\right)\left(1+b_{0} C\right) R_{01}\right)\left[\left(\left(1+a_{0} C\right)\left(1+b_{0} C\right) R_{0 v}\right)+D_{v}\right]\left[k_{1}+k_{v}-r_{T}\right]
\end{aligned}
$$

10.2. A2

$$
\boldsymbol{J}_{1}=\left(\begin{array}{cccccc}
\mu_{T} & 0 & b_{1} & 0 & \frac{-r_{1 m} T}{D_{T_{b}}} & 0 \\
0 & (h C+\alpha) & -\frac{k_{1} T_{1}}{1+a_{0} C_{1}} & 0 & 0 & 0 \\
0 & -\frac{N_{T} \alpha}{1+b_{0} C} & \mu_{v}-\frac{N_{m} \alpha_{m} k_{2} M}{1+b_{0} C} & 0 & 0 & 0 \\
0 & 0 & M\left(k_{2}-r_{2 m}\right) & \mu_{M} & -r_{1 m} M_{1} & 0 \\
0 & 0 & 0 & 0 & b_{2} & 0 \\
0 & 0 & \rho_{T} T_{1} C_{1} & 0 & 0 & \mu_{c}
\end{array}\right)
$$

where

$$
\begin{aligned}
& b_{1}=T\left(\frac{k_{v}}{T+B_{T}}-\frac{r_{T}}{D_{v}}-\frac{k_{1}}{1+a_{0} C}\right) \\
& b_{2}=\mu_{T_{b}}+k_{3} T+k_{4} M_{1}-K r_{T_{b}}
\end{aligned}
$$

The eigenvalues are calculated by solving the characteristic polynomial of the Jacobian matrix $\left|J_{1}-\lambda I\right|=0$ which can be expanded to become

$$
\left(\lambda-\mu_{T}\right)\left(\lambda-\mu_{c}\right)\left(\lambda-\mu_{M}\right)\left(\lambda-b_{2}\right)|(\lambda-a)(\lambda-d)-b c|=0
$$

where

$$
\begin{aligned}
& a=(h C+\alpha), \\
& b=\frac{N_{T} \alpha}{1+b_{0} C}, \\
& c=\frac{k_{1} T}{1+a_{0} C}, \\
& d=\mu_{v}-\frac{N_{m} \alpha k_{2} M}{1+b_{0} C} .
\end{aligned}
$$

\subsection{A3}

The Jacobian matrix evaluated at the virally infected endemic steady state is given by 


$$
\boldsymbol{J}_{2}=\left(\begin{array}{cccccc}
w_{1} & 0 & w_{2} & 0 & w_{3} & \frac{-k_{1}}{\left(1+a_{0} C_{2}\right)^{2}} \\
w_{4} & -((h C)+\alpha) & \frac{k_{1} T}{1+a_{0} C_{2}} & 0 & 0 & w_{5} \\
0 & w_{6} & w_{7} & \frac{N_{m} \alpha_{m} k_{2} V_{2}}{1+C_{0} C_{2}} & 0 & w_{8} \\
0 & 0 & M_{2}\left(r_{2 m}-k_{2}\right) & w_{9} & r_{1 m} M_{2} & 0 \\
0 & 0 & 0 & 0 & w_{10} & 0 \\
\rho_{T} V_{2} C_{2} & 0 & \rho_{T} T_{2} C_{2} & 0 & 0 & \rho_{T} V_{2}-\mu_{c}
\end{array}\right)
$$

where

$$
\begin{aligned}
& w_{1}=-\mu_{T}+r_{t}\left(\frac{V_{2}}{V_{2}+D_{v}}\right)+V_{2}\left(\frac{k_{v} B_{T}}{\left(T_{2}+B_{T}\right)^{2}}\right)-V_{2}\left(\frac{k_{1}}{1+a_{0} C_{2}}\right) \\
& w_{2}=\frac{r_{T} T_{2} D_{v}}{\left(V_{2}+D_{v}\right)^{2}}-T_{2}\left(\frac{k_{1}}{1+a_{0} C_{2}}\right)-T_{2}\left(\frac{k_{v}}{T_{2}+B_{T}}\right) \\
& w_{3}=\frac{r_{1 m} T_{2}}{D_{T_{b}}} \\
& w_{4}=\frac{k_{1} V_{2}}{1+a_{0} C_{2}} \\
& w_{5}=\frac{a_{0} k_{1} V_{2}}{\left(1+a_{0} C_{2}\right)^{2}}-h C_{2} \\
& w_{6}=\frac{N_{T} \alpha}{1+b_{0} C_{2}} \\
& w_{7}=\frac{N_{m} \alpha_{m} k_{2} M_{2}}{1+b_{0} C_{2}}-\mu_{v} \\
& w_{8}=\frac{b_{0}\left(N_{T} \alpha T_{2}^{*}+N_{m} \alpha_{m} k_{1} M_{2} V_{2}\right)}{\left(1+b_{0} C_{2}\right)^{2}} \\
& w_{9}=-\mu_{M}+V_{2}\left(r_{2 m}-k_{2}\right) \\
& w_{10}=r_{T_{b}} K-\mu_{T_{b}}-k_{3} T_{2}+k_{4} M_{2}
\end{aligned}
$$

10.4. A4

$$
\boldsymbol{J}_{3}=\left(\begin{array}{cccccc}
v_{1} & 0 & \frac{-r_{t} T}{D_{v}} & 0 & -\frac{r_{1 m} D_{T_{b}}}{\left(T_{b}+D_{T_{b}}\right)^{2}} & 0 \\
0 & -(h C+\alpha) & \frac{k_{1} T}{1+a_{0} C} & 0 & 0 & -h T^{*} \\
0 & \frac{N_{T} \alpha}{1+b_{0} C} & \frac{N_{m} \alpha_{m} k_{2} M}{1+b_{0} C}-\mu_{v} & 0 & 0 & \frac{N_{T} \alpha b_{0} T^{*}}{\left(1+b_{0} C\right)^{2}} \\
0 & 0 & M\left(r_{2 m}-k_{2}\right) & -\mu_{M}+r_{1 m} T_{b} & r_{1 m} M & 0 \\
-k_{5} T_{b} & 0 & 0 & -k_{4} T_{b} & v_{2} & 0 \\
0 & 0 & \rho_{T} T C & 0 & 0 & -\mu_{c}
\end{array}\right)
$$


where

$$
\begin{aligned}
& v_{1}=-\mu_{T}+r_{m}\left(\frac{T_{b}}{T_{b}+D_{T_{b}}}\right) \\
& v_{2}=r_{T_{b}}\left(K-2 T_{b}\right)-\mu_{T_{b}}-k_{3} T+k_{4} M
\end{aligned}
$$

the characteristic polynomial of the $\left|J_{3}-\lambda I\right|=0$ is given by

$\left|\begin{array}{cc}c-\lambda & f \\ d & g-\lambda\end{array}\right|[(a-\lambda)(m-\lambda)(c-\lambda)-j(a-\lambda)(i-\lambda)+b(m-\lambda)-b j]=0$

where

$$
\begin{aligned}
& a=-\mu_{T}+r_{m}\left(\frac{T_{b}}{T_{b}+D_{T_{b}}}\right), \\
& b=-k_{5} T_{b}, \\
& c=-(h C+\alpha), \\
& d=\frac{\alpha N_{T}}{1+b_{0} C}, \\
& e=\frac{-r_{t} T}{D_{v}}, \\
& f=\frac{k_{1} T}{1+a_{0} C}, \\
& g=w_{7}, \\
& h=M\left(r_{2 m}-k_{2}\right), \\
& i=\rho_{T} C T, \\
& j=-\mu_{M}+r_{1 m} T_{b}, \\
& k=-k_{4} T_{b}, \\
& m=r_{1 m}, \\
& n=r_{T_{b}}\left(K-2 T_{b}\right)-\mu_{T_{b}}-\left(k_{3} T+k_{4} M\right), \\
& p=-\mu_{c}
\end{aligned}
$$


Submit or recommend next manuscript to SCIRP and we will provide best service for you:

Accepting pre-submission inquiries through Email, Facebook, LinkedIn, Twitter, etc. A wide selection of journals (inclusive of 9 subjects, more than 200 journals)

Providing 24-hour high-quality service

User-friendly online submission system

Fair and swift peer-review system

Efficient typesetting and proofreading procedure

Display of the result of downloads and visits, as well as the number of cited articles

Maximum dissemination of your research work

Submit your manuscript at: http://papersubmission.scirp.org/

Orcontact 迆@scirp.org 